\title{
Nuevo paradigma, nuevo contexto institucional: el caso de la política farmacéutica
}

\author{
Laura Chaqués Bonafont
}

Resumen: El objetivo de este estudio es explicar cómo y por qué cambia la política farmacéutica española en la última década. En el se argumenta la relevancia de las instituciones como variables explicativa de la dinámica del cambio en las políticas siguiendo el esquema teórico planteado por el análisis de redes y la teoría del equilibrio interrumpido desarrollada por Baumgartner y Jones. La adopción de un nuevo paradigma en torno al medicamento -el uso racional del medicamento- no puede ser entendido únicamente como una respuesta a situaciones de crisis, cambios en el sistema político y/o la consolidación de ideas nuevas defendidas por activos emprendedores políticos. El análisis de la institucionalización de las relaciones de poder en torno a la política farmacéutica (o policy network) es importante para explicar por qué los cambios en la política farmacéutica se producen de forma gradual como resultado de una negociación entre el Estado con la industria farmacéutica y las farmacias. Asimismo, se argumenta cómo la adopción de un paradigma nuevo en la política farmacéutica, se produce de forma paralela a un cambio en la estructura institucional de la política. En 2008 la política farmacéutica se gestiona a través de una red más abierta y plural, en la que los expertos tienen mayor peso en la toma de decisiones.

Palabras clave: Redes de actores, política farmacéutica, agencias reguladoras, grupos de interés.

\section{INTRODUCCIÓN}

En 2008 el consumo de medicamentos sigue siendo uno de los aspectos centrales de la política sanitaria en la mayoría de países de la Unión Europea. El desarrollo tecnológico, el incremento del coste de la innovación del medicamento, el envejecimiento de la población, la universalización de la sanidad son factores que contribuyen a incrementar el gasto farmacéutico en la mayoría de los países desarrollados hasta alcanzar situaciones de crisis, lo que genera una intensificación en el nuevo milenio de lo que se ha denominado política de uso racional del medicamento. El de- 
sarrollo de este paradigma no es algo nuevo. La mayoría de países europeos ponen en práctica estas ideas en los años ochenta con el fin de generar un doble cambio, uno de carácter sanitario orientado a garantizar que los ciudadanos tengan acceso y utilicen de forma adecuada medicamentos y productos sanitarios de calidad, seguros y eficaces de acuerdo a sus necesidades clínicas; y otro de carácter económico orientado a garantizar la eficacia y eficiencia de la prestación farmacéutica, y por tanto a garantizar el acceso a los medicamentos al menor coste posible para el Estado.

La forma y la intensidad en que se han desarrollado estos objetivos es muy distinta entre países. En Gran Bretaña la primera lista negativa de medicamentos se aprueba en 1984, en Alemania los precios de referencia se introducen en 1989, y a principios de los noventa la mayoría de países de la UE ponen en práctica mecanismos para fomentar el consumo de medicamentos genéricos. Por el contrario, en España no se adoptan instrumentos nuevos para contener el gasto farmacéutico hasta los años noventa, obviando algunas de las iniciativas adoptadas por otros países de su entorno. Tras la aprobación de la Ley del medicamento en 1990, se aprueba la primera lista negativa de medicamentos en 1993 por el gobierno socialista, en la que sólo se excluyen medicamentos con utilidad terapéutica nula o inaceptable lo que tiene un impacto prácticamente nulo sobre el consumo público de medicamentos; la segunda lista negativa de medicamentos se aprueba en 1998 por el partido popular con prácticamente el mismo impacto; los precios de referencia se comienzan a aplicar en 1998 y los medicamentos genéricos no se regulan hasta 1996 a través de la Ley de acompañamiento de los presupuestos del Estado. En 2007, el consumo de medicamentos genéricos en España apenas supera el 10\% del mercado frente al 60\% en los países escandinavos (IMS, 2007).

La mayoría de estos instrumentos de control del consumo de medicamentos quedan regulados a través de la Ley 29/2006, de 26 de julio, de garantías y uso racional de los medicamentos y productos sanitarios que se aprueba en 2006 con el objetivo de intensificar la política de uso racional del medicamento. Esta ley, más que plantear ideas nuevas, adapta los principios ya establecidos en la Ley 25/1990, de 20 de diciembre a un contexto nuevo de globalización, integración europea y consolidación del proceso de descentralización política del Sistema Nacional de Salud a las Comunidades Autónomas. Esta estabilidad en la política de uso racional del medicamento en España; y el retraso en la implementación de los instrumentos de control del consumo hasta finales de los años noventa o principios del nuevo milenio puede explicarse parcialmente a través del trade-off existente entre la política de control del consumo frente a la política industrial farmacéutica (Lobo, 1992).

El sector farmacéutico es un sector clave para el fomento de la innovación y desarrollo tecnológico, básicamente porque sus actividades requieren de inversiones elevadas en I+D y ocupan a personal especializado en estas tareas. En España, el 18,8\% del gasto total y el $11 \%$ del empleo en I+D lo lleva a cabo la industria farmacéutica, a pesar de que la facturación de las empresas farmacéuticas suponen únicamente el 2,1\% de la industria (Seuba, 2009). Ello significa que la industria farmacéutica española 66 dedica casi el $7 \%$ de sus ventas a actividades de I+D frente al $0,8 \%$ del conjunto de la 
industria Española. En la actualidad apoyar al sector farmacéutico es un objetivo prioritario para cumplir con los objetivos del Plan Ingenio inspirados en el acuerdo Lisboa, que básicamente significa incrementar la inversión pública y privada en I+D hasta alcanzar el $2 \%$ del PIB y aumentar la participación empresarial hasta que financie el $55 \%$ del total de la inversión en I+D en 2010'. Este apoyo es especialmente importante en un contexto de desaceleración continuada de las inversiones de la industria farmacéutica española en $\mathrm{I}+\mathrm{D}^{2}$, proceso que se produce de forma paralela la desaceleración de la tasa de crecimiento del sector por debajo del crecimiento de la economía de forma continuada desde $2002^{3}$.

El sector farmacéutico también es uno de los ejes centrales de la política sanitaria tanto en lo relativo al acceso de los medicamentos, como al control del consumo sanitario. En la última década el consumo público de medicamentos crece a un ritmo anual superior al 8\% (Tabla 1). En total se consumen en España 14.250 millones de euros en medicamentos de los cuales el Estado paga casi el 75\% en 2007, un 4\% más que en 1995. El incremento del consumo público de medicamentos se explica por el incremento del consumo de pensionistas que consumen el $72,7 \%$ de los medicamentos con una subvención del 100\% sobre el precio; así como por el incremento continuado de los precios de los medicamentos más nuevos. Como consecuencia del incremento del gasto público farmacéutico, en la última década se intensifica la política de uso racional del medicamento cuyos objetivos, en la mayoría de ocasiones chocan por incompatibles con los objetivos de fomento al desarrollo de la industria farmacéutica innovadora.

\section{TABLA 1}

Evolución del consumo y gasto farmacéutico, 1995-2006 (millones de euros)

\begin{tabular}{|c|c|c|c|c|c|c|c|c|c|}
\hline Anual & $\begin{array}{c}\text { Mercado } \\
\text { total }\end{array}$ & $\begin{array}{c}\% \\
\text { Variación } \\
\text { anual }\end{array}$ & $\begin{array}{c}\text { Consumo } \\
\text { público total }\end{array}$ & $\begin{array}{c}\% \\
\text { Variación } \\
\text { anual }\end{array}$ & $\begin{array}{c}\text { Aportación } \\
\text { beneficiarios }\end{array}$ & $\begin{array}{c}\% \\
\text { Variación } \\
\text { anual }\end{array}$ & $\begin{array}{c}\text { Aportación } \\
\text { farmacéutica }\end{array}$ & $\begin{array}{c}\text { Gasto } \\
\text { farmacéutico }\end{array}$ & $\begin{array}{c}\% \\
\text { Variación } \\
\text { anual }\end{array}$ \\
\hline 1995 & 6.014 & 12 & $4.815,19$ & 12,1 & 426,17 & 8,3 & 70,67 & $4.318,36$ & 11,6 \\
\hline 1996 & 6.566 & 9,2 & $5.340,19$ & 10,9 & 453,53 & 6,4 & 72,3 & $4.814,37$ & 11,5 \\
\hline 1997 & 6.978 & 6,3 & $5.615,60$ & 5,2 & 460,38 & 1,5 & & $5.155,22$ & 7,10 \\
\hline 1998 & 7.658 & 9,7 & $6.174,72$ & 10 & 474,56 & 3,1 & & $5.700,16$ & 10,60 \\
\hline 1999 & 8.437 & 10,2 & $6.762,54$ & 9,5 & 494,69 & 4,2 & & $6.267,85$ & 10,00 \\
\hline 2000 & 9.115 & 8 & $7.319,94$ & 8,2 & 518,55 & 4,8 & 62,8 & $6.738,59$ & 7,5 \\
\hline
\end{tabular}

1 Estos objetivos quedan algo lejos de los objetivos fijados en Lisboa -aumentar el gasto en I+D hasta alcanzar el 3\% del PIB y conseguir que al menos dos terceras partes de este gasto provengan del sector privado-, pero son objetivos realistas que tienen en cuenta la realidad de la innovación en España. El gasto en I+D en España apenas supera el 1,1\% del PIB, porcentaje todavía muy lejos del 1,84\% del conjunto de la UE-27; del mismo modo la inversión privada en I+D en España es tan sólo de un $46 \%$ del total, frente al $74 \%$ de Japón, el $61 \%$ de EE UU o el $54 \%$ de la UE-27.

2 En 2006 las inversiones en I+D crecieron el 3,6\% respecto al año anterior -792 millones de euros-, cifra que contrasta con el $20 \%$ de crecimiento anual que se venía registrando en los últimos años.

3 De acuerdo con el informe del IMS (2007) este proceso de desaceleración de las ventas de medicamentos es una característica común a todos los países desarrollados. 


\section{TABLA 1 (cont.)}

Evolución del consumo y gasto farmacéutico, 1995-2006 (millones de euros)

\begin{tabular}{|c|c|c|c|c|c|c|c|c|c|}
\hline Anual & $\begin{array}{c}\text { Mercado } \\
\text { total }\end{array}$ & $\begin{array}{c}\% \\
\text { Variación } \\
\text { anual }\end{array}$ & $\begin{array}{c}\text { Consumo } \\
\text { público total }\end{array}$ & $\begin{array}{c}\% \\
\text { Variación } \\
\text { anual }\end{array}$ & $\begin{array}{c}\text { Aportación } \\
\text { beneficiarios }\end{array}$ & $\begin{array}{c}\% \\
\text { Variación } \\
\text { anual }\end{array}$ & $\begin{array}{c}\text { Aportación } \\
\text { farmacéutica }\end{array}$ & $\begin{array}{c}\text { Gasto } \\
\text { farmacéutico }\end{array}$ & $\begin{array}{c}\% \\
\text { Variación } \\
\text { anual }\end{array}$ \\
\hline 2001 & 9.990 & 9,6 & $8.019,35$ & 9,6 & 557,07 & 7,4 & 190,63 & $7.271,65$ & 7,9 \\
\hline 2002 & 10.949 & 9,6 & $8.839,03$ & 10,2 & 605,14 & 8,6 & 237,72 & $7.996,17$ & 10 \\
\hline 2003 & 12.207 & 11,5 & $9.948,54$ & 12,6 & 679,51 & 12,3 & 306,91 & $8.962,11$ & 12,1 \\
\hline 2004 & 12.974 & 6,3 & $10.514,00$ & 5,7 & 668,98 & $-1,6$ & 315,09 & $9.529,93$ & 6,3 \\
\hline 2005 & 13.580 & 4,7 & $11.129,16$ & 5,9 & 694,68 & 3,8 & 359,03 & $10.075,44$ & 5,7 \\
\hline 2006 & 14.250 & 4,9 & $11.787,15$ & 5,9 & 720,08 & 3,7 & 401,52 & $10.665,55$ & 5,9 \\
\hline
\end{tabular}

Fuente: Elaboración propia a partir de datos Ministerio de Sanidad y Consumo y Farmaindustria.

En este artículo se argumenta que para dar una explicación más completa al desarrollo de la política de uso racional del medicamento es necesario tener en cuenta, además de las características estructurales del mercado de medicamentos, las características del entramado institucional en el que se desarrolla la política o policy network. Los cambios en la estructura del mercado farmacéutico, el incremento continuado del gasto en medicamentos, unido a cambios institucionales como la intensificación del proceso de europeización, la agencificación (creación de la European Agency for the Evaluation of Medicinal Products -Emea- y la Agencia Española de Medicamentos -Agemed-), y la consolidación del proceso descentralización política abren ventanas de oportunidad política que en ocasiones son utilizadas para generar un cambio en la política. Estas oportunidades de cambio están condicionadas por la forma en que se estructuran las relaciones de poder entre los actores y por las reglas del juego que durante décadas han definido los objetivos y estrategias de esos actores en la política de uso racional del medicamento en España.

Otro de los objetivos de este artículo consiste en explicar la transformación gradual del marco institucional o red de actores en torno a la política farmacéutica, de una comunidad de actores cerrada dominada por los intereses empresariales hacia un modelo más abierto y plural en el que se da especial relevancia a los grupos de expertos como actor político (Tabla 2). Esta transformación se concreta en una fragmentación creciente en los intereses de la industria farmacéutica entre las empresas de genéricos y el resto de empresas del sector representadas por Farmaindustria. A su vez, la distribución de competencias y poder político dentro del Estado también se fragmenta como consecuencia de un triple proceso de delegación de poder político por parte del Ministerio de Sanidad: hacia la Unión Europea, hacia las Comunidades Autónomas y hacia la Agencia del Medicamento. Obviamente este traspaso de poder político es desigual y se lleva a cabo a través de procesos distintos, pero la idea básica es que la red de actores se fragmenta y esa fragmentación se produce de forma paralela a la consolidación del 68 uso racional del medicamento como paradigma en la política del medicamento. 


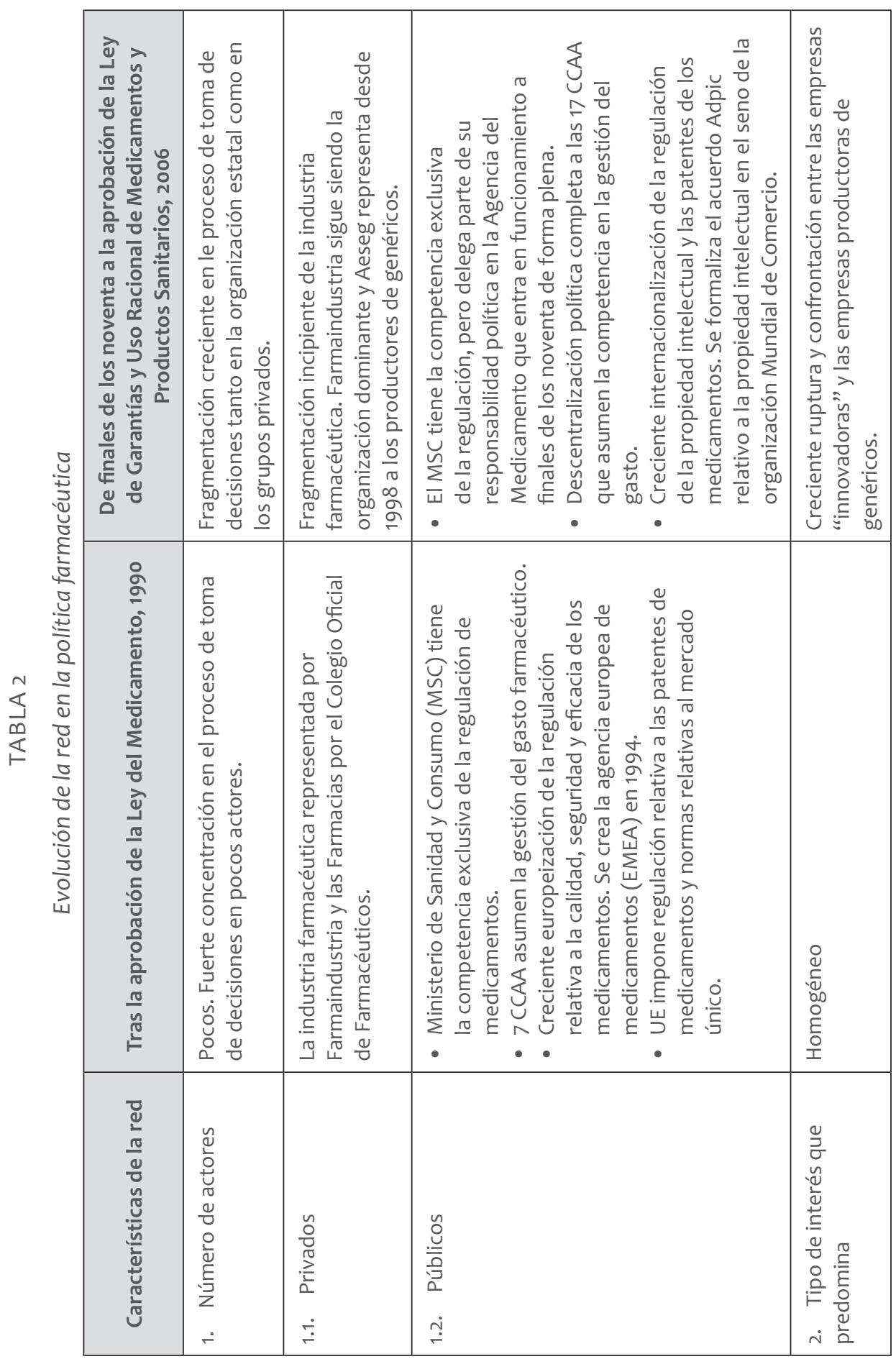




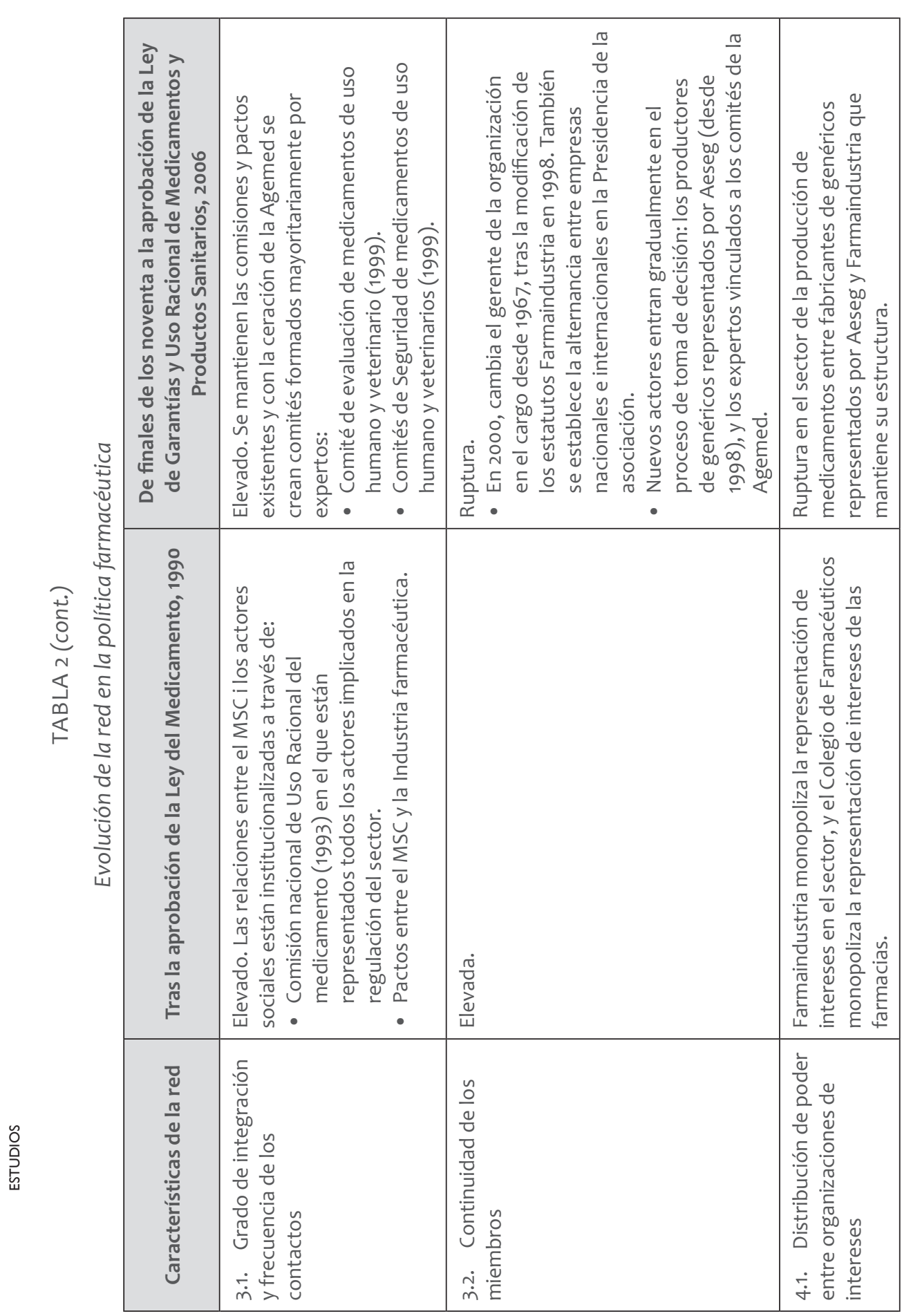


NUEVO PARADIGMA, NUEVO CONTEXTO INSTITUCIONAL: EL CASO DE LA POLÍTICA FARMACÉUTICA

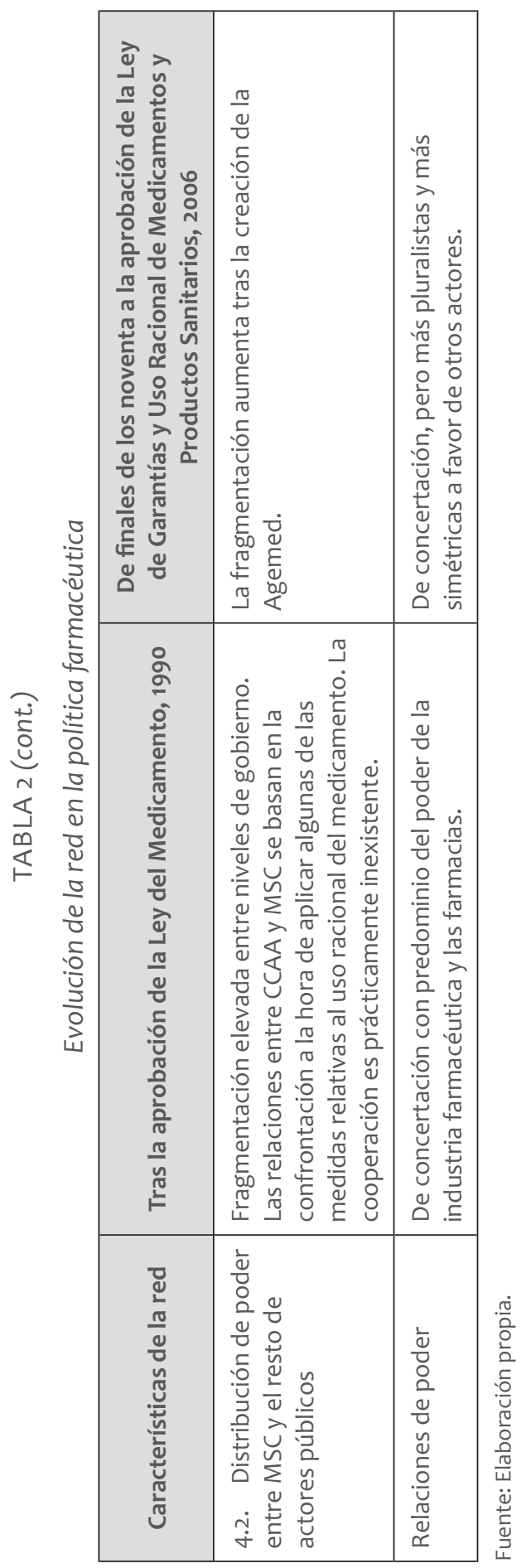


En definitiva, este artículo tiene como objetivo realizar una aportación al análisis de cómo las instituciones facilitan y limitan las oportunidades de cambio en las políticas a partir del análisis de la política de uso racional del medicamento. En el se plantean dos hipótesis generales. La primera sostiene que la existencia de redes de actores cerradas o comunidades políticas (Rhodes, 1990), facilita la estabilidad en las políticas y evita cambios radicales en el paradigma existente. La segunda sostiene que el cambio en la forma de entender y definir los objetivos de la política farmacéutica (o paradigma) se produce de forma paralela a un cambio en la estructura institucional (o policy network) en el que se desarrolla esa política. El cambio en el paradigma y en el marco institucional van unidos; si cambia uno, el otro también cambia. A través de este estudio de caso se argumenta pues la validez del modelo de cambio definido por Baumgartner y Jones (1993) como modelo del equilibrio interrumpido (o punctuated equilibrium), así como la relevancia del análisis de redes como marco analítico capaz de describir las diferentes formas de interacción entre los actores que participan en la negociación, decisión y puesta en práctica de una política.

Para explicar estas cuestiones el artículo se organiza en cuatro apartados. El primero discute las principales aportaciones teóricas y marcos analíticos existentes que analizan la interrelación entre cambio político y factores instituciones dentro del análisis de las políticas públicas. En el segundo apartado se analizan los cambios en la organización estatal y el proceso de triple delegación de competencias, y en el tercero se analiza el proceso de fragmentación de intereses en la industria farmacéutica. En el último apartado se presentan las principales conclusiones.

\section{MARCO ANALÍTICO}

Diferentes enfoques teóricos se centran en la interrelación entre factores institucionales y cambio político. En el neoinstitucionalismo histórico, se entiende que el proceso de elaboración de las políticas está dominado por largos periodos de estabilidad en los que los actores construyen conscientemente o reaccionan en contra de decisiones gubernamentales previas, con el fin de dar respuesta a problemas similares (True et al., 1999; Skocpol, 1985). Este proceso de cambio se produce siguiendo una dinámica incremental, en la que se redefinen los instrumentos (o algunos aspectos de estos instrumentos) de la política de acuerdo a un proceso de aprendizaje que generalmente está dominado por el Estado (Hall, 1993). Estos pequeños ajustes se producen de forma casi automática como reacción a cambios en el contexto o variables exógenas, manteniéndose intactos los principios y objetivos básicos de la políti-

o ca o paradigma político. El legado histórico y la estructura institucional son una fuente de estabilidad, que limitan las posibilidades de llevar a cabo un cambio radical en la política.

Estos periodos de estabilidad, en ocasiones, son alterados por cambios dramáticos promovidos por una combinación compleja de factores exógenos y endógenos

72 -como cambio tecnológico, el envejecimiento de la población, la universalización del 
sistema sanitario. Tal y como sugiere el modelo de las ventanas de oportunidad política, estos factores generan oportunidades para que se lleva a cabo un cambio en la política, aunque no necesariamente lo crean (Kingdon, 1995). De acuerdo con este modelo, el cambio en una política pública es el resultado de una compleja interacción entre tres procesos distintos que en principio siguen cursos distintos y no están relacionados entre si -el proceso relativo a los problemas, el proceso relativo a cambios en el sistema político, y el proceso relativo a la ideas disponibles-. Estos procesos se unen en algunas ocasiones, de forma poco o nada predecible, creando una oportunidad para un cambio en la política pública.

Para llevar a la práctica lo que Hall (1992) identifica como cambios de tercer nivel es necesario que se abra al menos una ventana de oportunidad política, bien porque un problema entra en crisis, bien porque se produce un cambio en el sistema político que es aprovechado por diferentes actores para introducir ideas nuevas respecto a la política. El hecho de que se abra una ventana de oportunidad política no implica necesariamente que se produzca ningún cambio; también es necesario que exista un emprendedor político capaz de identificar el momento más propicio para introducir con éxito ideas nuevas y redefinir el problema. Estos emprendedores políticos juegan un papel fundamental a la hora de unir estos procesos. "Mantienen su propuesta a la espera de un problema al que puedan aplicar su receta o una alteración en el flujo político, como puede ser un cambio administrativo que genere un clima receptivo para su propuesta" (Kingdon, 1995:195). El modelo de las ventanas de oportunidad política explica en parte el proceso de cambio en la política farmacéutica en las últimas tres décadas. Tal y como se argumenta en este artículo, las ventanas de oportunidad política y los emprendedores políticos son factores importantes para explicar los cambios en el proceso de elaboración de la política farmacéutica. Pero para dar una explicación completa de este proceso es necesario introducir como variable explicativa la estructura institucional del subsistema político o policy network.

Siguiendo el modelo del equilibrio interrumpido definido por Baumgartner y Jones (1993) en este artículo se argumenta que para llevar a cabo una redefinición de la política farmacéutica es preciso no sólo que se abran oportunidades para el cambio, sino también que se transforme la estructura institucional en el que se desarrolla la política. Jones y Baumgartner (2005) argumentan, a través del análisis de diferentes casos en Estados Unidos, que la forma en que se define una política (policy image) y el marco institucional en el que se desarrolla esa política se transforman de forma paralela: si cambia la estructura institucional, cambia la forma en que se define la política y viceversa. El cambio ocurre cuando un grupo se opone al estatus quo y trabaja para captar la atención y movilizar a actores que previamente mantienen una conducta pasiva respecto a la política en favor de sus propuestas (Baumgartner y Jones 1993, 2005). “A medida que la presión para el cambio se incrementa, es posible que la resistencia se imponga. Pero si quienes presionan son los suficientes, podrán llevar a cabo una intervención masiva en actores políticos e instituciones gubernamentales que hasta ahora habían permanecido al margen. Esto requiere, generalmente, un cambio sustancial en el apoyo de la forma en la que es definida una políti- 
ca. Una vez redefinida, o cuando se ponen de relieve nuevas dimensiones del debate, nuevos actores pasan a sentirse capacitados para ejercer su autoridad sobre lo que previamente se habían mantenido al margen. Estos nuevos actores pueden insistir en la redefinición de las normas y en cambiar la distribución del poder para reforzar nuevas estructuras institucionales, al mismo tiempo que las agencias e instituciones dominantes son obligadas a compartir su poder con grupos y agencias que ahora ganan legitimidad" (True et al., 1999: 101).

El análisis de redes (Richardson, 1979; Rhodes, 1990; Börzel, 1998; Jordana, 1995; Chaqués, 2004) como marco teórico, incrementa la capacidad explicativa tanto del modelo de las ventanas de oportunidad política como el modelo del equilibrio interrumpido. La idea de base es que la redefinición de las políticas, y por tanto la adopción de un paradigma nuevo, está condicionado (mediatizado) por la estructura de la red o policy network (Atkinson et al., 1989; Coleman, 1998). En función de las características de la red, la adopción de un paradigma nuevo es un proceso lento, pero de cambio asegurado y gestionado por pocos actores; o el resultado de un proceso abierto y politizado, en el que participan muchos actores con intereses contrapuestos en un contexto poco o nada institucionalizado (Marsh y Smith, 2000). Para analizar el impacto de la red de actores en el proceso de cambio de una política pública, como la política farmacéutica, es imprescindible definir unas variables que sirvan para describir diferentes tipos de redes, y sentar las bases desarrollar una clasificación, a partir de las cual definir hipótesis sobre las dinámicas de cambio asociadas a un tipo u otro de red (Chaqués, 2004). Con el fin de describir el policy network en la política farmacéutica, en este artículo se utiliza la clasificación ${ }^{4}$ desarrollada por Rhodes (1990) y por tanto se tienen en cuenta como variables explicativas: la distribución de poder entre actores, el tipo de intereses que predominan, el número de actores, la frecuencia de los contactos, el grado de institucionalización de las relaciones entre organizaciones públicas y privadas, así como la capacidad de cada una de estas organizaciones para definir sus propios objetivos y llevarlos a cabo de forma autónoma. Estas variables sirven para describir dos modelos contrapuestos -comunidad política y red en torno a problemas-.

Tal y como se argumenta en el resto del artículo, el caso de la política farmacéutica viene a corroborar que (1) para llevar a cabo un cambio de paradigma en la política es necesario que se produzca una alteración en la estructura institucional en la que se dirigen y gestionan aspectos clave de esa política; (2) siguiendo el esquema planteado por Jones y Baumgartner (2005) o Hall (1993) el cambio de paradigma en la política farmacéutica es el resultado de un debate politizado que se inicia -en los años ochenta-como resultado de situaciones de crisis -como el incremento en el gasto farmacéutico, o el incremento del coste de la innovación de medicamentos-, que cuestiona el paradigma existente. Una vez identificada la crisis se inicia un proceso de experimentación y debate que se intensifica en los años noventa, una vez se ha

4 En Chaqués (2004) se explican con detalle otras las clasificaciones que se han desarrollado hasta el momento en el análisis de redes. 
aprobado la ley del medicamento. Esta transformación es dirigida y gestionada por una comunidad de actores capaces de defender el status quo, evitar cambios radicales en los objetivos y prevenir cambios en la distribución de poder entre actores. En el siguiente apartado se explica cómo a partir de finales de los años noventa esta comunidad de actores cerrada se fragmenta de forma gradual lo que permite la consolidación de la política racional del medicamento como paradigma político.

\section{INSTITUCIONES Y REDES DE ACTORES: CAMBIOS EN LA ESTRUCTURA ESTATAL}

En 2008, el marco institucional en el que se desarrolla la política farmacéutica presenta un cambio substancial respecto a etapas anteriores. El proceso de elaboración de la política del medicamento está dirigido y gestionado por una comunidad de actores más abierta y plural; el número de actores que participa en la política aumenta; los intereses son más heterogéneos, coexistiendo diferentes formas de entender la política; las relaciones entre actores siguen siendo fluidas y permanentes, y se desarrollan en un marco formalmente institucionalizado, pero son más visibles y transparentes; la representación de los intereses de la industria farmacéutica se fragmentan, aunque siguen manteniendo muchas de las características que consolidan su poder de influencia; y la organización estatal se transforma de forma radical debido a un triple proceso de cambio: la descentralización política, la europeización y la agencificación. Este proceso de cambio de la organización estatal tiene una triple dimensión: (1) una de carácter vertical de delegación gradual de poder a otras instituciones para dirigir e implementar aspectos clave de la política del medicamento -básicamente la Unión Europea, y las Comunidades Autónomas-; (2) otra de carácter interno de delegación funcional a la Agencia Española del Medicamento y Productos Sanitarios (Agemed); y otra (3) horizontal de consolidación de la autonomía del Estado vis-à-vis actores privados.

En cuanto a la delegación vertical, la regulación básica de medicamentos tanto en relación a las medidas sanitarias de calidad, seguridad y eficacia de los medicamentos, como de los instrumentos de control del gasto es competencia del Gobierno central. El Ministerio de Sanidad, a través de la Dirección General de Farmacia, es quien tiene la responsabilidad de desarrollar la regulación de estas materias en todo el territorio, y las Comunidades Autónomas asumen la gestión del gasto farmacéutico a partir de este marco regulador común con un cierto margen de maniobra en cuanto a la intensidad y el ritmo de su aplicación. Este proceso de descentralización no se consolida hasta principios del nuevo milenio una vez se han transferido las competencias del Insalud de forma plena, lo que significa que durante casi dos décadas tan sólo siete CCAA tienen competencias en la gestión del gasto farmacéutico. La descentralización plena de la gestión del gasto farmacéutico no ha generado cambios importantes en las relaciones entre el gobierno central y las CCAA, las cuales siguen caracterizándose por la inexistencia de instituciones que garanticen la cooperación, negociación e intercambio de recursos entre ambos niveles de gobierno. El Consejo 
Interterritorial del Sistema Nacional de Salud, como institución básica de cooperación en cuestiones sanitarias, sigue sin consolidarse como instrumento al servicio del gobierno central y las CCAA para resolver conflictos y desarrollar una postura común respecto al uso racional del medicamento. Más que cooperar, cada CCAA desarrolla su política sin tener en cuenta las posibilidades de cooperación con el resto de CCAA.

En relación a la regulación farmacéutica impulsada por el gobierno central, las comunidades autónomas mantienen una postura crítica que en ocasiones ha resultado en una confrontación abierta con un fuerte impacto mediático. La primera de estas confrontaciones se produce durante la tramitación de la Ley del medicamento de 1990, en relación a la distribución de competencias respecto a las oficinas de farmacia, competencias que finalmente quedan atribuidas a las CCAA. Otras de las confrontaciones más intensas se produce tras la aprobación de la Ley del Medicamento, una vez el gobierno central decide excluir listas de medicamentos del Sistema Nacional de Salud. En 1998 dos CCAA (Andalucía y Navarra) deciden no aplicar esta regulación, asumiendo el coste de estos medicamentos a cargo del presupuesto autonómico. De todas formas, la oposición de las Comunidades Autónomas a la financiación selectiva de medicamentos no va acompañada de una política pro-activa orientada a definir una estrategia innovadora para controlar el gasto farmacéutico. Por el contrario, las CCAA asumen una postura reactiva, crítica y poco creativa respecto a la política de uso racional del medicamento desarrollada por el gobierno central. Sólo a finales de los años noventa algunas comunidades autónomas, como Cataluña y Andalucía, definen estrategias innovadoras para fomentar el uso de los medicamentos genéricos y el control de la prescripción por parte de los profesionales sanitarios. En cualquier caso, las CCAA son un actor clave en el proceso de elaboración de la política farmacéutica, que de forma gradual va adquiriendo protagonismo en la implementación de los instrumentos de control del consumo de medicamentos, lo que contribuye a la fragmentación del marco institucional que gobierna la política.

De forma paralela, el Gobierno Central delega competencias a la Unión Europea que se consolida como un nuevo escenario en el que organizaciones públicas y privadas interactúan entre si con el fin de influir en el proceso político. Al contrario que las CCAA, la UE asume el papel de actor innovador, que fomenta el cambio hacia una política de uso racional del medicamento. Además de promover la armonización en objetivos de carácter económico e industrial -eliminar las barreras al comercio, adoptar la patente de producto y la objetividad en la fijación de precios de los medicamentos-, la UE promueve el proceso de armonización de la regulación de la autorización

气 y registro de medicamentos con el fin de garantizar los mismos estándares de calidad, seguridad y eficacia de los medicamentos en toda la UE, y así eliminar el uso de estas regulaciones con fines proteccionistas. Este proceso de armonización se consolida con la creación en 1993 de la European Agency for the Evaluation of Medicinal Products (EMEA) como el máximo organismo evaluador comunitario tanto de los medicamentos que se introducen en el mercado como por aquellos ya comercializados, 76 controlados a través de un programa de farmacovigilancia. El cambio respecto eta- 
pas anteriores es que los dictámenes de la EMEA son vinculantes, a pesar de que la Agencia no tiene competencia sobre el precio o las condiciones de financiación de los medicamentos.

La creación de esta agencia pretende transformar el marco institucional de regulación para potenciar la competitividad de las empresas farmacéuticas, especialmente las grandes multinacionales. La EMEA contribuye a este objetivo reduciendo en parte las barreras existentes hasta 1995 que (1) obligan a las empresas a preparar la documentación necesaria y específica de cada país aumentando los recursos económicos y de tiempo necesarios para autorizar un medicamento en toda la UE (entre otros factores se obligaba a las empresas a pagar las tasas de registro cada vez que solicita la autorización); y que (2) retrasan la comercialización de un medicamento, lo que afecta tanto a las empresas porque se reduce el periodo de monopolio de la patente, como a la población en general al retrasar la entrada de un medicamento nuevo en el mercado. En definitiva, la EMEA es una institución nueva creada para (1) potenciar el mercado único de medicamentos; (2) la objetividad y transparencia en les actuaciones de los poderes públicos; y (3) eliminar las trabas burocráticas que impiden beneficiarse al máximo del poder de monopolio que otorgan las patentes, así como (4) potenciar la competitividad de uno de los sectores mas intensivos en I+D y con un valor estratégico elevado centrado en la investigación en biotecnología (Krapohl, 2004). Por último, la implantación del sistema Europeo de autorización de medicamentos también fomenta la competencia entre las diferentes Agencias del Medicamento de cada Estado miembro dado que cada laboratorio puede escoger libremente el Estado miembro a partir del cual quiere proyectar la comercialización de un medicamento. Eso significa que las empresas escogerán entre las agencias nacionales que efectúen la autorización y registro de forma más ágil y eficiente y con rigor científico suficiente para que sus dictámenes sean tenidos en cuenta por el resto de países de la UE. En este sentido, la creación de un marco regulador europeo crea incentivos para que los estados miembros transformen no sólo el contenido de la regulación sino también las estructuras que llevan acabo la regulación de la autorización y registro de medicamentos (Chaqués y Palau, 2007).

La AGEMED se crea en este contexto de europeización de la regulación del registro y autorización de medicamentos, con el objetivo de garantizar la objetividad y la eficiencia en la gestión de la regulación. El objetivo consiste en convertir a la Agencia del Medicamento en un referente técnico capaz de gestionar el control de la calidad, eficacia y seguridad de los medicamentos, y sus efectos adversos al margen de consideraciones e intereses partidistas, a partir de criterios de excelencia y rigor científico, y dando una imagen de cientificidad y rigor ante la opinión pública. Agilizar la autorización y registro de medicamentos también es un elemento clave para garantizar la competitividad de las empresas en un contexto de crisis de competitividad en el que el retraso en la entrada de un producto en el mercado puede significar la reducción del periodo de explotación de la patente. Además, la AGEMED proporciona asesoría científica y técnica en materia de evaluación de medicamentos; y participa de forma activa en la planificación, avaluación, supervisión y control de medicamentos que au- 
toriza la EMEA, así como las reuniones organizadas por instituciones internacionales -Unión Europea, Consejo de Europa, OCDE5-.

La Ley de garantías y uso racional de los medicamentos y productos sanitarios (2006) aumenta las competencias de la AGEMED al introducir como criterio para la fijación de precios la utilidad terapéutica de los medicamentos (criterio básico junto con el grado de innovación), criterio que deberá ser evaluado de forma periódica por la AGEMED, a través de un informe que ha de ser remito a la comisión de precios del Ministerio de Sanidad. Para llevar a cabo estas funciones la ley prevé la creación del Comité de evaluación de la utilidad terapéutica de nuevos medicamentos, como instrumento clave de la política de control del consumo de medicamentos. A pesar de que ninguna de estas propuestas ha sido desarrollada hasta el momento, se ha iniciado un proceso de cambio orientado a la delegación en la AGEMED de funciones relativas al uso racional del medicamento, cambio que se puede explicar a partir de (1) la voluntad de políticos y gobernantes de encontrar una salida política que permite evitar responsabilidades sobre cuestiones que pueden ser impopulares o tener un coste político elevado -como la financiación selectiva de medicamentos y su utilidad terapéuticay/o (2) la necesidad de delegar funciones complejas y con contenido técnico elevado a expertos en la materia que puedan garantizar objetividad, rigor técnico y transparencia, así como aumentar la credibilidad de las instituciones públicas en el desarrollo de estas funciones. En cualquier caso, la no aplicación de este cambio pone de manifiesto que la selección de medicamentos a partir de cualquier criterio es una cuestión políticamente controvertida, que implica un coste político elevado, que de momento ningún gobierno, ni institución quiere asumir de forma global (Chaqués, 2009).

Por último, la creación de la AGEMED también tiene como objetivo transformar las relaciones de la organización estatal vis-a-vis con los grupos sociales potenciando la participación de expertos en el proceso de elaboración de la política. Este cambio en las relaciones de poder queda reflejado en las funciones y estructura del consejo asesor (en el que 12 de los 18 miembros son expertos de reconocido prestigio) así como en la estructura de los diferentes comités consultivos que forman la AGEMED -Comité de Evaluación de Medicamentos de Uso Humano; Comité de Seguridad de Medicamentos de Uso Humano ${ }^{6}$; Comité de Evaluación de Medicamentos de Uso Veterinario; Comité de Seguridad de Medicamentos de Uso Veterinario-. La composición y funciones de estos comités reflejan la voluntad de crear escenarios institucionales estables para la discusión y análisis de problemas relativos a la regulación de los medicamentos, con carácter abierto y plural en el que participan miembros del Gobierno, la AGEMED, la industria farmacéutica, el colegio de médicos, el de veterina-

5 Hasta la aprobación de la AGEMED estas competencias son gestionadas por el Centro Nacional de Farmacobiología del Instituto Carlos III; la Subdirección General de Gestión de la tasa suplementaria de la cuota láctea del fondo español de garantía agraria (FEGA); la Subdirección General de Evaluación de medicamentos; y la Subdirección General de Control Farmacéutico (las dos últimas de la Dirección General de Farmacia) www.agemed.es.

6 Los dos primeros se crean en 1999 como resultado de la reordenación de las comisiones ya existentes (Comisión Nacional de Evaluación de Medicamentos y la Comisión Nacional de Farmacovigilancia). 
rios y las organizaciones de consumidores-, y las Comunidades Autónomas (que sólo participan en los comités de seguridad que tratan los temas de farmacovigilancia).

Este cambio en las relaciones de poder entre organizaciones públicas y privadas viene a concluir una de las aspiraciones centrales del Ministerio de Sanidad en el primer gobierno socialista: generar una ruptura con la herencia del franquismo y transformar el marco institucional en el que se venía desarrollando la política hacia un escenario más acorde con los intereses del gobierno, y menos atento a las demandas de los grupos de interés que tradicionalmente dominan la política.

\section{FRAGMENTACIÓN EN LA ORGANIZACIÓN DE INTERESES}

En 2008 existen dos organizaciones que representan los intereses de la industria farmacéutica. Farmaindustria es la organización más antigua -se crea en los años sesenta- y durante casi veinte años -de 1979 a 1998- monopoliza los intereses del sector farmacéutico en España. En la actualidad está formada por 212 empresas, lo que representa el $85 \%$ de las ventas de medicamentos de prescripción, algo menos del $10 \%$ respecto 1979 (Chaqués, 2002: 277). AEGEG es una organización relativamente nueva que se crea en 1998 con el fin de representar y defender los intereses de los laboratorios de especialidades farmacéuticas, compañías fabricantes de materias primas y principios activos farmacéuticos o empresas de desarrollo tecnológico cuya actividad principal se desarrolle en el campo de los genéricos ${ }^{7}$ En la actualidad, representa a 26 de los 177 laboratorios que comercializan alguna especialidad farmacéutica genérica lo que significa que sólo representa al $15 \%$ de los laboratorios. Sin embargo estas 26 empresas controlan el $85 \%$ del mercado de medicamentos genéricos. Tan sólo tres de las empresas afiliadas a Aeseg son también empresas afiliadas a Farmaindustria (Sandoz Farmaceutica, Farmaprojects, y Alter) y dos pertenecen a un grupo empresarial que a su vez pertenece a Farmaindustria (Merck genéricos y Phamagenus del Grupo J. Uriach). El grado de solapamiento es bajo (algo menos del 20\%) lo que pone de manifiesto la autonomía e independencia de ambas organizaciones a la hora de desarrollar sus actuaciones y defender sus intereses frente al gobierno. Formalmente, AESEG asume la representación de las empresas productoras de medicamentos genéricos mientras Farmaindustria representa a los productores de medicamentos y productos farmacéuticos globalmente.

Una de las características más interesantes en este proceso de fragmentación es que AESEG no asume la representación de las empresas de pequeña y mediana dimensión, de capital nacional que tradicionalmente se dedican a la copia "legal" de medicamentos innovados por las grandes empresas (Lobo, 1992). Es decir, AESEG no se crea como resultado de una ruptura o confrontación en el seno de Farmaindustria, sino como una respuesta a un cambio en la estructura del mercado de medicamentos. Farmaindustria sigue representando al grupo de pequeños laboratorios, los cua-

7 La información sobre esta organización puede consultarse a través de su página web: www. aeseg.es. 
les siguen liderados por laboratorios con una larga tradición en el mercado -Fardi-, y mantienen una cuota de poder importante en la organización, junto al grupo de laboratorios de capital nacional y potencial innovador -Almirall, Esteve-, y el grupo de empresas de carácter multinacional, de capital extranjero -Novartis o Pfizer-.Tan sólo dos de los laboratorios que tradicionalmente ocupan cargos de máxima responsabilidad en Farmaindustria entre las empresas de pequeña y mediana dimensión -Alter y Uriach- defienden sus intereses en ambas organizaciones, mientras el resto de laboratorios mantienen la misma estrategia de movilización que décadas atrás. Ello es debido básicamente a que una parte importante de la producción de genéricos ha sido capturada por empresas nacionales que tradicionalmente se dedican a otro tipo de actividades en el sector farmacéutico y que ven en el mercado de genéricos una oportunidad de negocio -Farmaprojects-, así como por empresas multinacionales que de forma paralela a su actividad en el campo de la innovación se dedican a la producción de este tipo de productos y ocupan una parte importante de este mercado en España -Sandoz o Merck por ejemplo-.

AESEG, como organización de intereses, tiene menos poder y capacidad de influencia que Farmaindustria por diferentes motivos. Por un lado Farmaindustria es una organización profesionalizada que durante décadas ha demostrado su capacidad para defender de forma eficaz los diferentes intereses del sector frente al Estado. La clave del éxito de esta organización puede explicarse en gran parte por la capacidad de mantener la cohesión interna y garantizar que los conflictos entre los tres grupos de empresas del sector se resuelven a través de la negociación y búsqueda del consenso en los órganos políticos de la organización. Evitar la visibilidad de los conflictos y garantizar el monopolio de la representación es uno de los factores que más ha contribuido a mantener una organización fuerte capaz de negociar frente al gobierno aspectos clave de la política del medicamento. Asimismo, la capacidad de Farmaindustria para aglutinar en una única organización a la mayoría de laboratorios farmacéuticos garantiza que sea reconocida y legitimada por parte de los diferentes gobiernos como interlocutor válido en el proceso de elaboración de la política.

Por otro lado, Farmaindustria representa a un sector estratégico desde un punto de vista industrial y tecnológico, lo que garantiza una mayor sensibilidad por parte del Estado para responder a las demandas del sector y más en concreto a las grandes empresas de capital nacional. Este grupo de empresas asumen el compromiso de desarrollar actividades de I+D en el territorio español y a cambio el Estado se compromete a responder con especial intensidad a sus demandas en relación al gasto farmacéutico. Uno de los indicadores para medir el poder de este grupo de empresas es la evolución de la composición de los órganos de gobierno de Farmaindustria. A pesar de las modificación de los Estatutos de Farmaindustria en 1997, las empresas de gran dimensión y de capital nacional siguen teniendo un grado de representatividad más elevado que las empresas de capital extranjero. Las empresas de capital extranjero -básicamente grandes multinacionales del sector- que operan en España concentran la mayoría de las ventas del sector farmacéutico, pero comparten a partes iguales el 80 control de la organización con las empresas de capital nacional (Tabla 3). De hecho, 
no es hasta la renovación de los estatutos en 1997 cuando se establece el equilibrio de poder entre empresas de capital nacional y extranjero en un 50\%, y no es hasta 2001 cuando una empresa no nacional asume la presidencia de la organización.

TABLA 3

Principales laboratorios en España (2006)

\begin{tabular}{|c|c|c|c|}
\hline \multicolumn{1}{|c|}{ Puesto en el ranking } & $\begin{array}{c}\text { Ventas } \\
\text { (millones } \\
\text { de dólares) }\end{array}$ & $\begin{array}{c}\% \\
\text { Crecimiento } \\
\text { constante }\end{array}$ & $\begin{array}{c}\% \\
\text { Cuota } \\
\text { de mercado }\end{array}$ \\
\hline 1. Pfizer & 926,3 & 1,3 & 7,8 \\
\hline 2. Novartis & 730 & 11,2 & 6,1 \\
\hline 3. Sanofi-Aventis & 685,7 & 6,1 & 5,7 \\
\hline 4. Almirall & 648,7 & 1,8 & 5,4 \\
\hline 5. Glaxo SmithKline & 590,8 & 2,5 & 4,9 \\
\hline 6. AstraZéneca & 517,2 & 14,7 & 4,3 \\
\hline 7. Merck \& Co. & 492,4 & 6,5 & 4,1 \\
\hline 8. Johnson \& Jonhson & 484,1 & 8,8 & 3,1 \\
\hline 9. Esteve & 390,9 & 2,8 & 3,1 \\
\hline 10. Bayer & 373,4 & 0,5 & 48,9 \\
\hline Total 'top ten' & $5.839,3$ & 5,4 & 100 \\
\hline Total España & $11.940,8$ & 6,2 & \\
\hline
\end{tabular}

Fuente: IMS Health (2007).

El valor estratégico de la industria farmacéutica como sector líder en innovación es uno de los elementos clave que determina el poder de Farmaindustria a la hora de negociar frente al Estado aspectos clave de la política del medicamento. Este poder se consolida e institucionaliza a través de la participación de Farmaindustria en las instituciones formales en las que se deciden aspectos clave de la política farmacéutica. Farmaindustria sigue manteniendo una posición de privilegio frente al resto de actores sociales gracias a su participación en la negociación de los pactos frente al Estado, así como en los comités (de la AGEMED) en los que se deciden y gestionan aspectos clave relacionados con la política farmacéutica.

La capacidad de AESEG para presionar al sistema e influir en la política del medicamento es limitada en un contexto institucional cerrado (o policy community) dominado por Farmaindustria como organización que representa los intereses del sector. AESEG no participa en los pactos, ni en los comités de la agencia española del medicamento; dispone de una organización con menos recursos económicos, humanos y de información que Farmaindustria, y representa a los intereses de un segmento del mercado de medicamentos todavía muy reducido en relación al mercado de medica- 
mentos total. En 2007 el número de especialidades farmacéuticas aprobadas por la Agencia Española del Medicamento es de 3.313 (139 principios activos) que absorben algo más del $7 \%$ del Mercado farmacéutico en valores y el $14 \%$ en unidades. Estos porcentajes se sitúan muy lejos de la media europea que está en torno al 30-35\% y muy lejos de la media de Estados Unidos en torno al 50\% o los países escandinavos donde el mercado de genéricos se sitúa cerca del 60\% (Chaqués, 2009).

Sin embargo, en los últimos cinco años las empresas productoras de genéricos han visto reforzado su papel tanto en el escenario político como en el mercado debido a dos factores: (1) la voluntad política por parte de los gobiernos para fortalecer y renovar la política de uso racional de medicamentos y (2) el cambio de tendencia en el mercado caracterizado por la creciente exposición a los genéricos en clases estratégicas de medicamentos. El crecimiento de las ventas de medicamentos genéricos es superior al de los productos de marca en todos los países de nuestro entorno, y esa tendencia queda reforzada por el hecho de que entre 2008 y 2013, el 24\% del mercado de medicamentos, que equivale a 165,2 billones de dólares perderán la patente (IMS, 2007). En España el mercado que quedará expuesto se estima en 4,5 billones de dólares (algo más de $22 \%$ del mercado) y eso afectará a la mayoría de áreas terapéuticas, aunque con mayor intensidad a los medicamentos para tratar enfermedades cardiovasculares, gastroentereología y asma. La pérdida de peso específico de los medicamentos más innovadores queda reforzada por la reducción del número de lanzamientos de nuevos productos en el mercado. En 2007 el número de nuevas moléculas fue de 27, casi la mitad respecto a los medicamentos nuevos lanzados en 1997 (49 productos).

Este cambio en la tendencia del mercado se produce de forma paralela a la aprobación de la Ley 29/2006, de 26 de julio, de garantías y uso racional de los medicamentos y productos sanitarios. Por un lado, a través de esta ley se introduce la utilidad terapéutica de los medicamentos como criterio para justificar la financiación selectiva y no indiscriminada de medicamentos; así como la modificación del sistema de precios de referencia con el fin de garantizar el ahorro al Sistema Nacional de Salud y a su vez asegurar la previsibilidad, la estabilidad y la gradualidad en el impacto para la industria farmacéutica, afectando a todos los medicamentos en fase de madurez en el mercado. Por otro lado, la nueva ley también elimina los obstáculos que dificultan una mayor presencia de los medicamentos genéricos en el mercado, con el fin de equiparar la situación española con la de otros países de nuestro entorno ${ }^{8}$, y clarifica aspectos clave de la regulación de estos productos en España, regulación que se desarrolla de forma escalonada desde 1996.

Por último, la Ley 29/2006 también introduce cambios significativos en las reglas del juego que gobiernan la política farmacéutica y más en concentro en la política de

8 En relación a los medicamentos genéricos el cambio más relevante es el referente a la Cláusula Bolar. A partir de 2006 las empresas que quieran fabricar genéricos podrán iniciar las fases de investigación antes de que la patente del medicamento de marca haya caducado. Por tanto, si la empresa de genéricos realiza con éxito sus investigaciones podrá comercializar un producto genérico inmediatamente después de que la patente haya caducado. 
pactos entre el Ministerio de Sanidad y Consumo y la industria farmacéutica. En la disposición adicional sexta (1) se fija como criterio básico para realizar las aportaciones al Sistema Nacional de Salud por parte de los laboratorios el volumen de ventas de los laboratorios ${ }^{9}$ (excluyendo por ejemplo el pago de una cantidad fija); y (2) se define el carácter finalista de la aportación de forma que los recursos que se obtienen con el pacto se deben destinar a: la investigación en el ámbito de la biomedicina, al desarrollo de políticas de cohesión sanitaria y programas de formación para facultativos médicos y farmacéuticos y de educación sanitaria de la población, y para favorecer el uso racional de medicamentos. La definición de estos ámbitos de financiación es muy genérica lo que sigue otorgando un cierto margen de maniobra al Ministerio de Sanidad y Consumo y el Consejo Interterritorial del Sistema Nacional de Salud, los cuales mantienen su capacidad para definir la distribución de los recursos obtenidos a través de los pactos con la industria.

En cualquier caso, estos cambios establecen una ruptura importante en el marco regulador de la política farmacéutica. Durante décadas los pactos o conciertos con la industria farmacéutica se configuran como el instrumento clave en la política de control del gasto farmacéutico. Los conciertos como instrumentos para controlar el consumo de medicamentos son menos efectivos que los precios de referencia o el copago, pero su aplicación conlleva más ventajas tanto para la industria, como el Estado. El coste político asociado a la mayoría de los instrumentos de control del gasto (como la exclusión de medicamentos de la financiación pública o incrementar el co-pago) son elevados -imponen costes concentrados y generan beneficios difusos entre la población-. Por el contrario a través de estos conciertos el Ministerio de Sanidad obtiene recursos económicos de los actores privados (generalmente un descuento definido como un porcentaje de las ventas) que no tiene carácter finalista (y por tanto puede utilizar para financiar aquellas partidas que se considere oportuno en cada momento), a través de una negociación relativamente fácil, evitando el coste político de otros instrumentos de control del gasto. De la misma forma la industria farmacéutica prefiere este mecanismo de control, que le da un poder de negociación elevado para imponer sus demandas (como posponer la implementación instrumentos como los precios de referencia o los medicamentos genéricos) a cambio de una pago fijo o porcentaje de las ventas, que en muchos casos es fácil de compensar a través de estrategias comerciales de las empresas. Los cambios que se introducen con la nueva ley en principio sientan las bases para que la política de uso racional del medicamento esté menos sujeta a una negociación con los actores privados, y crea incentivos para potenciar otros instrumentos de control del gasto como los medicamentos genéricos o los precios de referencia (Chaqués y Palau, 2009).

9 Los laboratorios deben ingresar con carácter cuatrimestral las cantidades que resulten de aplicar sobre su volumen cuatrimestral de ventas a través de dicha receta, un porcentaje que oscila entre un mínimo del 1,5\% (hasta ventas de tres millones de euros) y un máximo del 5\% (ventas mayores a los 300 millones de euros. Estas cuantías quedan reducidas si la empresa dedica recursos a I+D dentro de la acción Profarma, y esta reducción varía en función de si la empresa esta clasificada como aceptable, buena, muy buena excelente (entre un 5 y $25 \%)$. 


\section{CONCLUSIONES}

La política farmacéutica, como estudio de caso, corrobora la tesis de Baumgartner y Jones $(1993,2005)$ sobre el proceso de cambio en las políticas públicas. El cambio en el contenido de la política del medicamento requiere un cambio en la forma en que se deciden y negocian aspectos clave de la regulación farmacéutica (o policy network). En 2008 la estructura institucional a través de la cual se dirigen y gestionan los objetivos básicos de la política farmacéutica se convierte en una estructura más abierta y plural en la que los grupos de expertos, al menos formalmente, tienen más capacidad de intervención en relación a etapas anteriores. Esta transformación en las relaciones de poder entre organizaciones públicas y privadas se caracteriza por la creciente fragmentación del poder tanto dentro del Estado como en la organización de intereses del sector farmacéutico. Dentro de la organización estatal, este cambio tiene una triple dimensión: de carácter vertical de transferencia de poder hacia las CCAA y la UE; de carácter interno de delegación de poder a la AGEMED; y de carácter horizontal de consolidación de la autonomía del Estado vís-á-vís los grupos sociales. En el caso de la organización de intereses la fragmentación se produce como consecuencia de los cambios en el mercado de medicamentos, y la regulación relativa al uso racional del medicamento de 2006. Los productores de medicamentos genéricos ganan terreno tanto en el ámbito económico y político aunque sólo sea de forma incipiente; y crean una organización propia para defender sus intereses cuestionando la hegemonía que durante décadas ha mantenido Farmaindustria como organización que representa los intereses de la industria del medicamento.

Estos cambios se consolidan con la aprobación de la Ley de garantías y uso racional de los medicamentos y productos sanitarios de 2006. A través de esta Ley se redefinen las reglas del juego dentro del sector farmacéutico y se introducen incentivos nuevos en el sistema de pactos entre el Ministerio de Sanidad y la industria farmacéutica que en principio deberían generan una mayor eficiencia tanto a nivel económico -fortaleciendo las actividades de I+D- como en el control del consumo farmacéutico -potenciando el consumo de medicamentos genéricos a través de la cláusula Bolar; o introduciendo la utilidad terapéutica de los medicamentos como criterio para la fijación de precios-. Por último, estos cambios en el contenido de la política se producen de forma paralela al cambio en el policy network, cambio que se lleva a cabo a lo largo de más de dos décadas como resultado de un proceso de adaptación gradual en el que el Ministerio de Sanidad, la industria farmacéutica y las farmacias ceden una parte de su poder a favor de otros actores. La gradualidad de este proceso es explicada por las características del policy network, y del marco regulador existente que crea incentivos para mantener el status quo, y evitar cambios dramáticos en la regulación.

\section{REFERENCIAS BIBLIOGRÁFICAS}

Atkinson, M. y Coleman, W.D. (1989), 'Strong States and Weak States: sectoral policy networks in advanced capitalist economies', British Journal of Political Science, 19(1): 47-67. 
Baumgartner, F. y Jones, B. (1993), Agenda and Instability in American Politics. Chicago: University of Chicago Press.

Börzel, T.A. (1998), “Organizing Babylon: On the different conceptions of policy networks”, Public Administration, 76: 253-273.

Chaqués, L. (2002), Estructura y política farmacéutica. Madrid: Centro de Investigaciones Sociológicas.

Chaqués, L. (2004), Redes de Políticas públicas. Madrid: Centro de Investigaciones Sociológicas.

Chaqués, L. (2009), “Fragmentación, judicialización, e internacionalización de la organización de intereses en el sector farmacéutico", en X. Seuba, ed., Salud Pública y Patentes Farmacéuticas. Cuestiones de Economía, Política y Derecho, Barcelona: Javier Bosch.

Chaqués, L. y Palau, A. (2007), Agències Reguladores Independents en l'àmbit Social. Anàlisis Comparativa del Sector Farmacèutic i el Sector de Seguretat Alimentària. Escola d'Administració pública de Catalunya.

Chaqués, L. y Palau, A. (2009), “The Dynamics of Policy Change: A Comparative Analysis of the Food Safety and Pharmaceutical Policy in Spain”, Journal of Public Policy, 29 (en prensa).

Coleman, W. D. (1998), “From protected development to market liberalism: paradigm change in agriculture”, Journal of European Public Policy, 5 (4): 632-651.

Hall, P. A. (1993), "Policy Paradigms, Social Learning and the State”, Comparative Politics, 25: 275-96.

Jones, B. y Baumgartner, F. (2005), The Politics of Attention. Chicago: University of Chicago.

Jordana, J. (1995), “El análisis de los policy networks: una nueva perspectiva sobre la relación entre políticas públicas y Estado”, Gestión y Análisis de Políticas Públicas, 3.

Kingdon, J.W. (1995), Agendas, Alternatives, and Public Policies. Nueva York: Harper Collins College Publishers.

Krapohl, S. (2004), “Credible Commitment in Non-Independent Regulatory Agencies: A Comparative Analysis of the European Agencies for Pharmaceuticals and Foodstuffs", European Law Journal, 10(5): 518-538.

Lobo, F. (1992), Medicamentos: Política y Economía. Barcelona: Masson. 
Marsh, D. y Smith, M. (2000), “Understanding policy networks: Towards a dialectical approach”, Political Studies 48 (1): 4-21.

Mossialos, E y Oliver, A. (2005), “An Overview of Pharmaceutical Policy in four Countries: France, Germany, the Netherlands and the United Kingdon”, International Journal of Health Planning and Management, 20: 291-306.

Rhodes, R.A.W. (1990), 'Policy networks. A British perspective', Journal of Theoretical Politics, 2(3): 1-26.

Richardson, J. y Jordan, A. G. (1979), Governing Under Pressure. Oxford: Martin Robertson.

Rothstein, B. (1996), “Political Institutions: An Overview”, en R. E. Goodin y H. D. Klingemann, eds., A New Handbook of Political Science. Oxford: Oxford University Press.

Seuba X. (ed.) (2009), Salud Pública y Patentes Farmacéuticas. Cuestiones de Economía, Política y Derecho. Barcelona: Javier Bosch.

Skocpol, T. (1985), "Bringing the State Back In: Strategies of Analysis in Current Research”, en P. Evans, D. Rueschemeyer y T. Skocpol, eds., Bringing the Sate Back In. New York: Cambridge University Press.

True, J. L., Jones, B.D. y Baumgartner, F.R. (1999), “Punctuated-equilibrium Theory: Explaining Stability and Change in American Policymaking”, en P.A. Sabatier, ed., Theories of the policy process. Oxford: Westview Press. 